\title{
Chiral corrections to the Roper mass ${ }^{1}$
}

\author{
B. Borasoy ${ }^{2 a}$, P. C. Bruns ${ }^{3 a}$, U.-G. Meißner ${ }^{4 a, b}$, R. Lewis ${ }^{5 c}$ \\ ${ }^{a}$ Helmholtz-Institut für Strahlen- und Kernphysik (Theorie) \\ Universität Bonn, Nußallee 14-16, D-53115 Bonn, Germany \\ ${ }^{b}$ Institut für Kernphysik (Theorie), Forschungszentrum Jülich \\ D-52425 Jülich, Germany \\ ${ }^{c}$ Department of Physics, University of Regina, Regina, Saskatchewan, Canada, S4S 0A2
}

\begin{abstract}
We analyze the quark mass dependence of the Roper mass to one-loop order in relativistic baryon chiral perturbation theory. The loop integrals are evaluated using infrared regularization which preserves chiral symmetry and establishes a chiral counting scheme. The derived chiral expansion of the Roper mass may prove useful for chiral extrapolations of lattice data. For couplings of natural size the quark mass dependence of the Roper mass is similar to the one of the nucleon.
\end{abstract}

PACS: $\quad$ 12.38.Gc, 12.39.Fe, 12.40.Yx

Keywords: Lattice QCD calculations, chiral Lagrangians, hadron mass models and calculations

\footnotetext{
${ }^{1}$ This research is part of the EU Integrated Infrastructure Initiative Hadron Physics Project under contract number RII3-CT-2004-506078. Work supported in part by DFG (SFB/TR 16, "Subnuclear Structure of Matter", and BO 1481/6-1) and by Canada's NSERC and CRC programs.

2 email: borasoy@itkp.uni-bonn.de

3 email: bruns@itkp.uni-bonn.de

${ }^{4}$ email: meissner@itkp.uni-bonn.de

${ }^{5}$ email: randy.lewis@uregina.ca
} 


\section{Introduction}

Understanding the (ir)regularities of the light quark baryon spectrum poses an important challenge for lattice QCD. In particular, the first even-parity excited state of the nucleon, the Roper $N^{*}$ (1440) (from here on called the Roper) is very intriguing - it is lighter than the first oddparity nucleon excitation, the $S_{11}(1535)$, and also has a significant branching ratio into two pions. Recent lattice studies, see e.g. [1-7], have not offered a clear picture about the nucleon resonance spectrum. In particular, lattice QCD operates at unphysical quark (pion) masses and thus a chiral extrapolation is needed to connect these data to the physical world. The findings of Ref. [2] indicate a rapid cross over of the first positive and negative excited nucleon states close to the chiral limit. No such level switching is e.g. found in [4], possibly related to the fact that the simulations were performed at quark masses too far away from the chiral regime. It should also be noted that so far very simple chiral extrapolation functions have been employed in most approaches, e.g., a linear extrapolation in the quark masses, thus $\sim m_{\pi}^{2}$ (with $m_{\pi}$ the pion mass), was applied in [7]. It is therefore important to provide the lattice practitioners with improved chiral extrapolation functions. This is the aim of this manuscript. We consider the Roper mass (the real part of the Roper self-energy) to one-loop in baryon chiral perturbation theory. More precisely, we employ an extension of the infrared regularization method of Ref. [8] and study the pion mass dependence as a function of the various low-energy (coupling) constants that appear in the expression. We refrain from analyzing the existing lattice data-our results apply to full QCD and not to one of the various approximations to QCD employed in the lattice studies.

Our manuscript is organized as follows. In Sec. 2, we display the effective chiral Lagrangian underlying our calculation. The chiral corrections to the Roper mass are calculated in Sec. 3. Sec. 4 contains our results and the discussion thereof.

\section{Effective Lagrangian}

We will calculate chiral corrections to the Roper mass up to one-loop order. Since the Roper is the first even-parity excited state of the nucleon, the construction of the chiral $\mathrm{SU}(2)$ effective Lagrangian follows standard procedures, see e.g. [9]. The effective Lagrangian relevant for our calculation is (see also Ref. [10])

$$
\mathcal{L}=\mathcal{L}_{0}+\mathcal{L}_{R}+\mathcal{L}_{N R}
$$

with the free part

$$
\mathcal{L}_{0}=i \bar{N} \gamma_{\mu} D^{\mu} N-M_{N} \bar{N} N+i \bar{R} \gamma_{\mu} D^{\mu} R-M_{R} \bar{R} R
$$

where $N, R$ are nucleon and Roper fields, respectively, and $M_{N}, M_{R}$ the corresponding baryon masses in the chiral limit. $D_{\mu}$ is the chiral covariant derivative, for our purpose we can set $D_{\mu}=\partial_{\mu}$, see e.g. [9] for definitions. The pion-Roper coupling is given to leading chiral order by

$$
\mathcal{L}_{R}^{(1)}=\frac{1}{2} g_{R} \bar{R} \gamma_{\mu} \gamma_{5} u^{\mu} R
$$

with an unknown coupling $g_{R}$ and the superscript denotes the chiral order. The pion fields are collected in $u_{\mu}=-\partial_{\mu} \boldsymbol{\pi} / f_{\pi}+\mathcal{O}\left(\boldsymbol{\pi}^{3}\right)$, where $f_{\pi}$ is the pion decay constant in the chiral limit. 
At next-to-leading order, the relevant terms in $\mathcal{L}_{R}$ are (we work in the isospin limit $m_{u}=m_{d}$ and neglect electromagnetism)

$$
\mathcal{L}_{R}^{(2)}=c_{1}^{*}\left\langle\chi_{+}\right\rangle \bar{R} R-\frac{c_{2}^{*}}{8 M_{R}^{2}} \bar{R}\left(\left\langle u_{\mu} u_{\nu}\right\rangle\left\{D^{\mu}, D^{\nu}\right\}+\text { h.c. }\right) R+\frac{c_{3}^{*}}{2}\left\langle u_{\mu} u^{\mu}\right\rangle \bar{R} R
$$

where $\chi_{+}$is proportional to the pion mass and induces explicit chiral symmetry breaking. Further, \langle\rangle denotes the trace in flavor space. For a complete one loop calculation we also need the fourth order effective Lagrangian, more precisely, the term

$$
\mathcal{L}_{R}^{(4)}=-\frac{e_{1}^{*}}{16}\left\langle\chi_{+}\right\rangle^{2} \bar{R} R
$$

The interaction piece between nucleons and the Roper reads

$$
\mathcal{L}_{N R}^{(1)}=\frac{1}{2} g_{N R} \bar{R} \gamma_{\mu} \gamma_{5} u^{\mu} N+\text { h.c. }
$$

The coupling $g_{N R}$ can be determined from the strong decays of the resonance $R$, its actual value is given below. In principle a term of the form

$$
i \lambda_{1} \bar{R} \gamma_{\mu} D^{\mu} N-\lambda_{2} \bar{R} N+\text { h.c. }
$$

is possible, but applying the equations of motion removes the first term (and its hermitian conjugate) such that we are left with the terms $\bar{R} N$ and $\bar{N} R$. These terms induce mixing between the nucleon and Roper fields, but diagonalization of the $N-R$ mass matrix does not lead to new operator structures and its effect can be completely absorbed into the couplings already present in the Lagrangian. We can thus safely work with the Lagrangian in Eq. (10). A complete one-loop calculation involves tree graphs with insertion of chiral dimension two and four and one-loop graphs with at most one insertion from $\mathcal{L}_{R}^{(2)}$.

\section{Chiral corrections to the Roper mass}

We are now in the position to work out the various contributions to the Roper mass. The loop diagrams are evaluated making use of (an extension of) the infrared regularization (IR) method [8]. As we will see, the IR scheme is suited for the study of systems with one light mass scale $m_{\pi}$ and two heavy mass scales $M_{N}, M_{R}$ with $M_{N}^{2} \ll M_{R}^{2}$. In the real world, we have $M_{R}^{2} / M_{N}^{2} \simeq 2.4$, so that this condition is approximately fulfilled.

1. Tree level: Only the $c_{1}^{*}$ and $e_{1}^{*}$ terms contribute to the self-energy at tree level

$$
\Sigma_{R}^{\text {tree }}=-4 c_{1}^{*} m_{\pi}^{2}+e_{1}^{*} m_{\pi}^{4} .
$$

These terms could be absorbed into $M_{R}$, but since we are interested in the explicit dependence on the pion (quark) mass, we must keep them. The first term is the leading order contribution to the nucleon-Roper $\sigma$-term.

2. Pion-nucleon loop: This is the only new structure compared e.g. to the calculation of the nucleon self-energy, see Fig. 1. We extend here the method of Ref. [11] developed for IR with vector mesons. Consider first the fundamental scalar integral at one-loop order in $d$ dimensions 

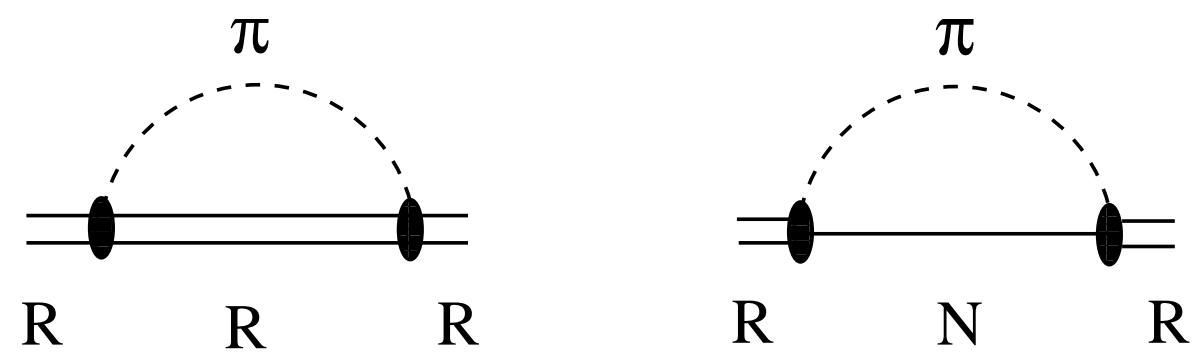

Figure 1: One-loop self-energy graphs of the Roper $(\mathrm{R})$ with intermediate Roper-pion $(\pi)$ and nucleon (N)-pion states, respectively.

with an intermediate pion-nucleon pair and external momentum $p$

$$
I_{\pi N}\left(p^{2}\right)=\int \frac{d^{d} l}{(2 \pi)^{d}} \frac{i}{\left[l^{2}-m_{\pi}^{2}+i \epsilon\right]\left[(p+l)^{2}-M_{N}^{2}+i \epsilon\right]} .
$$

Employing standard Feynman parameterization leads to

$$
I_{\pi N}\left(p^{2}\right)=-\frac{\Gamma\left(2-\frac{d}{2}\right)}{(4 \pi)^{d / 2}} M_{N}^{d-4} \int_{0}^{1} d z\left(\beta\left[z-x_{+}\right]\left[z-x_{-}\right]\right)^{\frac{d}{2}-2}
$$

in terms of the parameters

$$
x_{ \pm}=\frac{\alpha+\beta-1}{2 \beta}\left(1 \pm \sqrt{1-\frac{4 \alpha \beta}{(\alpha+\beta-1)^{2}}}\right), \quad \alpha=\frac{m_{\pi}^{2}}{M_{N}^{2}}, \quad \beta=\frac{p^{2}}{M_{N}^{2}} .
$$

In the following, we will restrict ourselves to the kinematical region $p^{2} \gg\left(M_{N}+m_{\pi}\right)^{2}$ which is equivalent to

$$
\frac{4 \alpha \beta}{(\alpha+\beta-1)^{2}} \ll 1
$$

This implies that in the chiral limit $\alpha \rightarrow 0$ the mass difference $p^{2}-M_{N}^{2}$ remains finite and does not tend towards zero as in the standard case of IR. This constraint is clearly satisfied for values $p^{2} \approx M_{R}^{2}$ close to the Roper mass. It is also consistent with resonance decoupling in the chiral limit [12]. The nucleon propagator is thus counted as zeroth chiral order for momenta $p^{2} \approx M_{R}^{2}$. Setting $\beta=M_{R}^{2} / M_{N}^{2}$ one obtains the small parameter

$$
\frac{4 \alpha \beta}{(\alpha+\beta-1)^{2}} \approx \frac{1}{9}
$$

which indicates a fast convergence of the expansion of the loop integral Eq. (9) in powers of the pion mass. Expansion of $x_{ \pm}$in $\alpha$ leads to

$$
\begin{aligned}
& x_{+}=\frac{\beta-1}{\beta}-\frac{\alpha}{\beta(\beta-1)}-\frac{\alpha^{2}}{(\beta-1)^{3}}+\mathcal{O}\left(\alpha^{3}\right), \\
& x_{-}=\frac{\alpha}{\beta-1}+\frac{\alpha^{2}}{(\beta-1)^{3}}+\mathcal{O}\left(\alpha^{3}\right),
\end{aligned}
$$


where $x_{-}=\mathcal{O}(\alpha)$ and $x_{+}=\mathcal{O}(1)$. We now divide the parameter integral of Eq. (10) into three parts

$$
I_{\pi N}=-\frac{\Gamma\left(2-\frac{d}{2}\right)}{(4 \pi)^{d / 2}} M_{N}^{d-4}\left(I_{\pi N}^{(1)}+I_{\pi N}^{(2)}+I_{\pi N}^{(3)}\right)
$$

with

$$
\begin{aligned}
& I_{\pi N}^{(1)}\left(p^{2}\right)=\int_{0}^{x_{-}} d z\left(\beta\left[z-x_{+}\right]\left[z-x_{-}\right]\right)^{\frac{d}{2}-2} \\
& I_{\pi N}^{(2)}\left(p^{2}\right)=\int_{x_{-}}^{x_{+}} d z\left(\beta\left[z-x_{+}\right]\left[z-x_{-}\right]\right)^{\frac{d}{2}-2} \\
& I_{\pi N}^{(3)}\left(p^{2}\right)=\int_{x_{+}}^{1} d z\left(\beta\left[z-x_{+}\right]\left[z-x_{-}\right]\right)^{\frac{d}{2}-2} .
\end{aligned}
$$

Note that $0<x_{-}<x_{+}<1$. The first integral $I_{\pi N}^{(1)}$ can be rewritten as

$$
I_{\pi N}^{(1)}\left(p^{2}\right)=\left(x_{-}\right)^{d / 2-1} \int_{0}^{1} d y\left(\beta\left[x_{+}-x_{-}(1-y)\right]\right)^{\frac{d}{2}-2} y^{\frac{d}{2}-2} .
$$

Expansion of the integrand in powers of $x_{-} \sim \mathcal{O}(\alpha)$ and interchanging summation with integration leads to

$$
I_{\pi N}^{(1)}\left(p^{2}\right)=\frac{2}{d-2}\left(x_{-}\right)^{d / 2-1}\left(\beta x_{+}\right)^{d / 2-2}+\mathcal{O}\left(\alpha^{d / 2}\right),
$$

where the leading term is of order $\mathcal{O}\left(\alpha^{d / 2-1}\right)$ and thus conserves power counting. The integral $I_{\pi N}^{(1)}$ contains only fractional powers of $\alpha$ and contributes to the infrared singular part. The remaining two integrals, on the other hand, are regular in $\alpha$. For $I_{\pi N}^{(2)}$ one has

$$
I_{\pi N}^{(2)}\left(p^{2}\right)=(-\beta)^{d / 2-2} \int_{x_{-}}^{x_{+}} d z\left(\left[x_{+}-z\right]\left[z-x_{-}\right]\right)^{\frac{d}{2}-2}
$$

where $\beta$ has a small positive imaginary piece. Integration yields

$$
I_{\pi N}^{(2)}\left(p^{2}\right)=(-\beta)^{d / 2-2}\left(x_{+}-x_{-}\right)^{d-3} \frac{\left(\Gamma\left(\frac{d}{2}-1\right)\right)^{2}}{\Gamma(d-2)} .
$$

Obviously, this expression is expandable in powers of $x_{-}$. The integral $I_{\pi N}^{(2)}$ is complex and does not conserve power counting. It contributes only to the regular part and will be omitted for our purposes. More precisely, the imaginary part does not contribute to the resonance mass, while the real part can be absorbed into the couplings of the effective Lagrangian at the on-shell momentum $p^{2}=M_{R}^{2}$. In the third integral,

$$
I_{\pi N}^{(3)}\left(p^{2}\right)=\int_{x_{+}}^{1} d z\left(\beta\left[z-x_{+}\right]\left[z-x_{-}\right]\right)^{\frac{d}{2}-2},
$$

one can expand the integrand directly in powers of $x_{-}$. The expansion coefficients of this series are integrals of the type $(r \in \mathbb{R})$

$$
\int_{x_{+}}^{1} d z\left(z-x_{+}\right)^{\frac{d}{2}-2} z^{r}=\left(1-x_{+}\right)^{d / 2-1} \int_{0}^{1} d w(1-w)^{\frac{d}{2}-2}\left(1+w\left(x_{+}-1\right)\right)^{r} .
$$


Since $1-x_{+}$remains finite in the chiral limit these integrals also contribute only to the regular part and can be absorbed into the couplings of the Lagrangian at momentum $p^{2}=M_{R}^{2}$. Therefore, the infrared singular part which stems from small values of the Feynman parameter $z$ is entirely contained in $I_{\pi N}^{(1)}$ and we can restrict ourselves to the integral

$$
\begin{aligned}
\left(I_{\pi N}\right)_{I R} & =-\frac{\Gamma\left(2-\frac{d}{2}\right)}{(4 \pi)^{d / 2}} M_{N}^{d-4} \int_{0}^{x_{-}} d z\left(\beta\left[x_{+}-z\right]\left[x_{-}-z\right]\right)^{\frac{d}{2}-2} \\
& =-\frac{M_{N}^{d-4}}{16 \pi^{2}}\left\{x_{-}\left(\frac{2}{4-d}+\ln 4 \pi-\gamma_{E}+1\right)-\int_{0}^{x_{-}} d z \ln \left(\beta\left[x_{+}-z\right]\left[x_{-}-z\right]\right)\right\}
\end{aligned}
$$

Expansion in $\alpha$ leads to

$$
\left(I_{\pi N}\right)_{I R}=\left(2 L+\frac{1}{16 \pi^{2}} \ln \left(\frac{m_{\pi}^{2}}{M_{R}^{2}}\right)\right)\left(\frac{\alpha}{\beta-1}+\frac{\alpha^{2}}{(\beta-1)^{3}}\right)-\frac{1}{32 \pi^{2}} \frac{\alpha^{2} \beta}{(\beta-1)^{3}}+\mathcal{O}\left(m_{\pi}^{6}\right)
$$

with

$$
L=\frac{M_{R}^{d-4}}{16 \pi^{2}}\left\{\frac{1}{d-4}-\frac{1}{2}\left[\ln 4 \pi-\gamma_{E}+1\right]\right\},
$$

and $\gamma_{E}$ is the Euler-Mascheroni constant. We have chosen the regularization scale to be $M_{R}$. In fact, the same result for the infrared part can be obtained by expanding the baryon propagator in the loop integral (a method first used in Ref. [13])

$$
\int \frac{d^{d} l}{(2 \pi)^{d}} \frac{i}{\left[l^{2}-m_{\pi}^{2}\right]\left[p^{2}+2 p \cdot l+l^{2}-M_{N}^{2}\right]} .
$$

Counting the loop momentum as $l \sim \mathcal{O}\left(m_{\pi}\right)$ and taking $p^{2} \gg\left(M_{N}+m_{\pi}\right)^{2}$, one obtains

$$
\frac{1}{p^{2}-M_{N}^{2}} \int \frac{d^{d} l}{(2 \pi)^{d}} \frac{i}{l^{2}-m_{\pi}^{2}}\left(1-\frac{2 p \cdot l+l^{2}}{p^{2}-M_{N}^{2}}+\frac{\left(2 p \cdot l+l^{2}\right)^{2}}{\left(p^{2}-M_{N}^{2}\right)^{2}}+\ldots\right)
$$

which reproduces the result of Eq. (24) after interchanging summation and integration.

After investigating the scalar loop integral one readily obtains the infrared singular part of the full one-loop self-energy diagram with an intermediate pion-nucleon pair (see Fig. II)

$$
\begin{aligned}
\left(\Sigma_{N}(\not p)\right)_{I R}= & i \frac{3 g_{N R}^{2}}{4 f_{\pi}^{2}} \int_{I R} \frac{d^{d} l}{(2 \pi)^{d}} \frac{l\left(p+l-M_{N}\right) l}{\left[l^{2}-m_{\pi}^{2}\right]\left[(p+l)^{2}-M_{N}^{2}\right]} \\
=-\frac{3 g_{N R}^{2}}{4 f_{\pi}^{2}}(\not p & {\left[\frac{m_{\pi}^{4}}{32 \pi^{2}\left(p^{2}-M_{N}^{2}\right)} \ln \left(\frac{m_{\pi}^{2}}{M_{R}^{2}}\right)+\frac{m_{\pi}^{4}}{64 \pi^{2}\left(p^{2}-M_{N}^{2}\right)}\right] } \\
& \left.+\frac{M_{N} m_{\pi}^{4}}{16 \pi^{2}\left(p^{2}-M_{N}^{2}\right)} \ln \left(\frac{m_{\pi}^{2}}{M_{R}^{2}}\right)\right),
\end{aligned}
$$

where we have only displayed the finite pieces. The term proportional to $L$ has been absorbed into the counter terms. Evaluating the integral at $\not p=M_{R}$ yields

$$
\left(\Sigma_{N}\left(M_{R}\right)\right)_{I R}=-\frac{3 g_{N R}^{2}}{256 \pi^{2} f_{\pi}^{2}\left(M_{R}^{2}-M_{N}^{2}\right)} m_{\pi}^{4}\left[\left(2 M_{R}+4 M_{N}\right) \ln \left(\frac{m_{\pi}^{2}}{M_{R}^{2}}\right)+M_{R}\right] .
$$


Note that this expression preserves both power counting and chiral symmetry.

3. Pion-Roper loop: This corresponds to the standard IR case and is immediately obtained from the result in [8] by replacing $M_{N}$ by $M_{R}$ (see Fig. 11),

$$
\left(\Sigma_{R}\left(M_{R}\right)\right)_{I R}=-\frac{3 g_{R}^{2}}{32 \pi f_{\pi}^{2}} m_{\pi}^{3}\left[1+\frac{m_{\pi}}{2 \pi M_{R}}+\frac{m_{\pi}}{2 \pi M_{R}} \ln \left(\frac{m_{\pi}^{2}}{M_{R}^{2}}\right)\right]+\mathcal{O}\left(m_{\pi}^{5}\right)
$$

Again, power counting and chiral symmetry are maintained for the IR singular part of this diagram.

4. Tadpoles: The tadpoles with vertices from $\mathcal{L}_{R}^{(2)}$ yield

$$
\Sigma_{R}^{\mathrm{tad}}=\left(6 c_{1}^{*}-\frac{3}{4} c_{2}^{*}-3 c_{3}^{*}\right) \frac{m_{\pi}^{4}}{16 \pi^{2} f_{\pi}^{2}} \ln \left(\frac{m_{\pi}^{2}}{M_{R}^{2}}\right)+\frac{3}{128 \pi^{2} f_{\pi}^{2}} c_{2}^{*} m_{\pi}^{4} .
$$

Again, this result agrees with the one for the nucleon by proper substitution of the LECs and baryon masses.

5. Total Roper self-energy: Putting all these pieces together, we obtain the following one-loop correction to the Roper mass

$$
\delta M_{R}^{(1-\text { loop })}=\left(\Sigma_{N}\left(M_{R}\right)\right)_{I R}+\left(\Sigma_{R}\left(M_{R}\right)\right)_{I R}+\Sigma_{R}^{\text {tad }}+\Sigma_{R}^{\text {tree }}
$$

in terms of the renormalized couplings $c_{1}^{*}, e_{1}^{*}$ and the renormalized mass $M_{R}$ for which we use the same notation.

We have not explicitly considered loops with a $\Delta(1232)$-pion pair ${ }^{6}$. If one treats the $\Delta$ on the same footing as the nucleon field, the contribution will be of the type in Eq. (29) and amounts to a renormalization of $g_{N R}$ and $e_{1}^{*}$. However, one must keep in mind that the convergence of the corresponding chiral series is not as good as for the nucleon due to the smaller mass difference $M_{R}^{2}-M_{\Delta}^{2}$. If, on the other hand, the Delta mass is considered to be of the same size as the Roper mass, the loop contribution will be similar to the result in Eq. (30) and leads to a modification of $g_{R}$ and the couplings $c_{i}^{*}, e_{1}^{*}$. In both scenarios, the effects of the $\Delta \pi$ loop can be absorbed into a redefinition of the couplings. Since their values are not fixed, we will vary them within certain regions, see Sec. 4, such that the inclusion of the $\Delta$ resonance will not alter any of our conclusions. For a treatment of the $\Delta$ in infrared regularization see, e.g., Refs. [15-17]. We also stress that the explicit inclusion of the $\Delta$ can lead to a complicated three-small-scales problem (the pion mass and - if considered small - the Roper-Delta and the Delta-nucleon splitting) that requires theoretical tools that have not yet been developed for baryon chiral perturbation theory.

\section{Quark mass dependence of the Roper mass}

Before analyzing the pion mass dependence of the Roper mass, we must collect information on the couplings $g_{R}, g_{N R}$ and the LECs $c_{i}^{*}(i=1,2,3)$, and $e_{1}^{*}$. One obtains $g_{N R}=0.3 \ldots 0.4$ from a fit to the branching ratio of the Roper into one pion and a nucleon which is in agreement

\footnotetext{
${ }^{6}$ Note that in the Jülich coupled-channels approach, the Roper is dynamically generated with an important $\pi \Delta$ component besides the $\sigma N$ one [14].
} 
with the relation $g_{N R}=\sqrt{R} g_{A} / 2$, where $g_{A}$ is the axial-vector coupling of the nucleon and $\sqrt{R}=0.53 \pm 0.04$ (for details, see Ref. [18]). For $g_{R}$ the naive quark model predicts $g_{R}=g_{A}$, and we set here $g_{R}=1.0$ so that $g_{A}$ and $g_{R}$ are roughly of the same size, see also [19].

To leading order in the chiral expansion, the LEC $c_{1}^{*}$ measures the $\sigma$-term in the Roper state and it is thus bounded from above by the value of the pion-nucleon $\sigma$-term. This means $\left|c_{1}^{*}\right| \lesssim 1 \mathrm{GeV}^{-1}$. More realistically, a natural value for $c_{1}^{*}$ would be around $-0.5 \mathrm{GeV}^{-1}$ because $\sigma$-terms are expected to become smaller with the resonance excitation energy (see also the related discussion on the $\pi \Delta \sigma$-term in Refs. [16,17,20,21]). The sign of $c_{1}^{*}$ should be negative since the quark masses contribute positively to the hadron masses. The nucleon LECs $c_{2}$ and $c_{3}$ are much enhanced compared to the natural values $\left|c_{i}\right| \lesssim 1 \mathrm{GeV}^{-1}$ because of the nearby and strongly coupled delta resonance [22]. This is not expected to be the case for the Roper resonance. Consequently, the LECs $c_{2,3}^{*}$ can be bounded conservatively by $\pm 1 \mathrm{GeV}^{-1}$. The pion decay constant in the chiral limit is taken to be $f_{\pi}=87 \mathrm{MeV}$ [23].

In Fig. 2 an estimated range for the pion mass dependence of the Roper mass is presented by taking the extreme values for $c_{2,3}^{*}$ and $e_{1}^{*}$, while keeping $c_{1}^{*}=-0.5 \mathrm{GeV}^{-1}, g_{N R}=0.35, g_{R}=1$ fixed. The masses of the baryons in the chiral limit are taken to be $M_{N}=0.885 \mathrm{GeV}$ [24] and $M_{R}=1.4 \mathrm{GeV}$, respectively. The dash-dotted curve is obtained by setting the couplings $c_{2,3}^{*}, e_{1}^{*}$ all to zero, and exhibits up to an offset a similar quark mass dependence as the nucleon result (dotted curve, taken from Ref. [24]). It should be emphasized, however, that the one-loop formula cannot be trusted for pion masses much beyond $350 \mathrm{MeV}$. For similar results for the nucleon mass, see also Ref. [25].

In the numerical calculation we have employed the pion decay constant in the chiral limit, $f_{\pi}=87 \mathrm{MeV}$. However, we could have equally well used the physical pion decay constant, $F_{\pi}=92.4 \mathrm{MeV}$, as the difference in the chiral expansions appears either at chiral order $\mathcal{O}\left(m_{\pi}^{6}\right)$ in Eqs. (29, 31) or at order $\mathcal{O}\left(m_{\pi}^{5}\right)$ for the Roper-pion loop which is beyond the accuracy of the present investigation. The numerical results for these contributions would change by the amount of $f_{\pi}^{2} / F_{\pi}^{2} \approx 0.89$ and do not lead to significant changes in the results. Stated differently, the replacement of $f_{\pi}$ by $F_{\pi}$ in these formulae induces a correction due to the quark mass expansion of $F_{\pi}$

$$
F_{\pi}=f_{\pi}\left(1+\frac{m_{\pi}^{2}}{16 \pi^{2} f_{\pi}^{2}} \bar{l}_{4}+\mathcal{O}\left(m_{\pi}^{4}\right)\right)
$$

with $\bar{l}_{4}=4.33$. The modifications at leading order are then

$$
-\frac{3 g_{N R}^{2}}{2048 \pi^{4} F_{\pi}^{4}\left(M_{R}^{2}-M_{N}^{2}\right)} \bar{l}_{4} m_{\pi}^{6}\left[\left(2 M_{R}+4 M_{N}\right) \ln \left(\frac{m_{\pi}^{2}}{M_{R}^{2}}\right)+M_{R}\right]
$$

for the nucleon-pion loop,

$$
-\frac{3 g_{R}^{2}}{256 \pi^{3} F_{\pi}^{4}} \bar{l}_{4} m_{\pi}^{5}
$$

for the Roper-pion loop, and

$$
\left(6 c_{1}^{*}-\frac{3}{4} c_{2}^{*}-3 c_{3}^{*}\right) \bar{l}_{4} \frac{m_{\pi}^{6}}{128 \pi^{4} F_{\pi}^{4}} \ln \left(\frac{m_{\pi}^{2}}{M_{R}^{2}}\right)+\frac{3}{1024 \pi^{4} F_{\pi}^{4}} c_{2}^{*} \bar{l}_{4} m_{\pi}^{6} .
$$

for the tadpoles. These corrections at higher chiral orders are indeed small and can be safely neglected for small pion masses. In fact, the variations induced by these corrections are within the band for $M_{R}\left(m_{\pi}^{2}\right)$ given in Fig. 2 , 


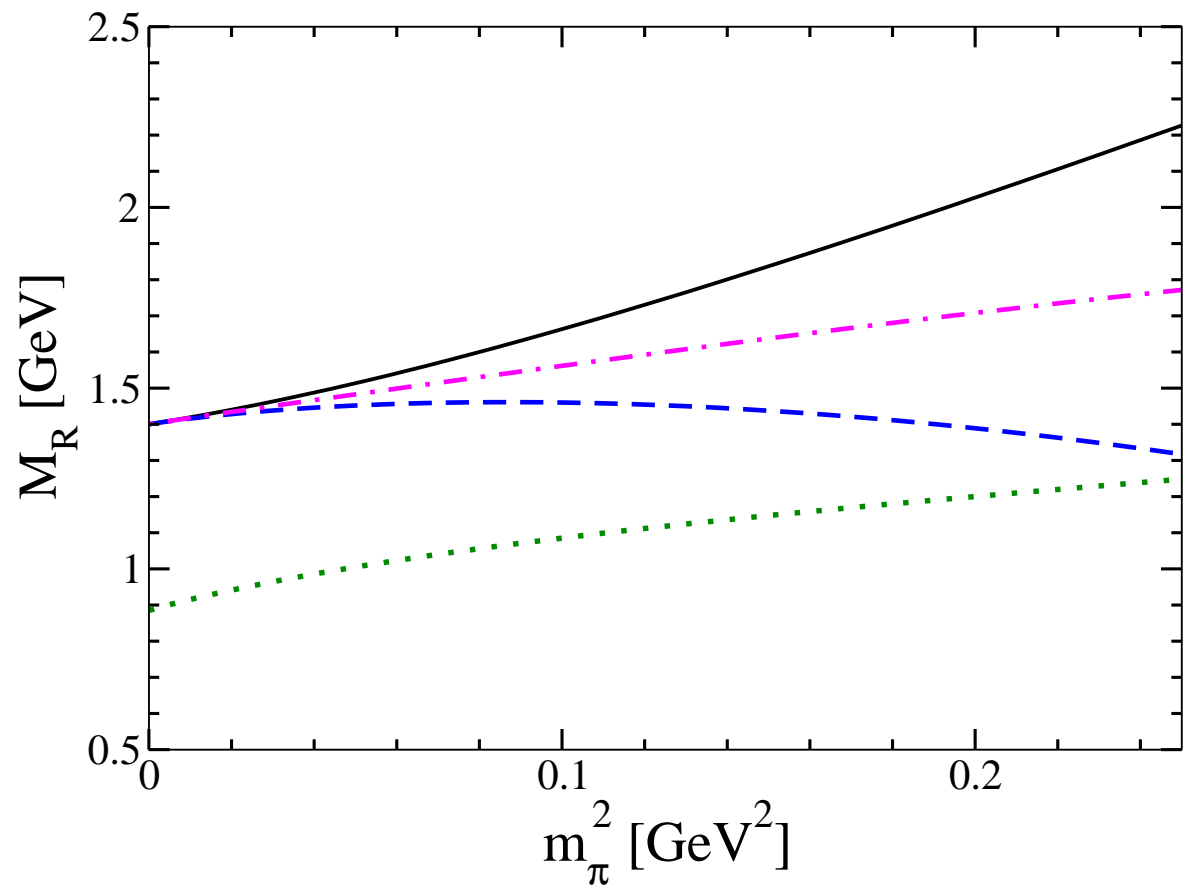

Figure 2: Quark mass dependence of the Roper mass for different parameter sets $c_{1}^{*}=$ $-0.5, c_{2,3}^{*}, e_{1}^{*}$. The $c_{i}$ are in units of $\mathrm{GeV}^{-1}$ and $e_{1}$ is given in $\mathrm{GeV}^{-3}$. and couplings $g_{R}=1.0, g_{N R}=0.35$. The solid curve corresponds to $c_{2}^{*}=1.0, c_{3}^{*}=1.0, e_{1}^{*}=0.5$, the dashed one to $c_{2}^{*}=-1.0, c_{3}^{*}=-1.0, e_{1}^{*}=-0.5$ and the dot-dashed one to $c_{2}^{*}=c_{3}^{*}=e_{1}^{*}=0$. The dotted curve represents the quark mass dependence of the nucleon, see Ref. [24]. The values of the corresponding LECs are: $c_{1}=-0.9, c_{2}=3.2, c_{3}=-3.45, e_{1}=-1.0$.

In this work, we have calculated the chiral corrections to the Roper mass to one-loop order. The approach is based on an extension of infrared regularization which allows for the unambiguous isolation of the infrared singular part of the loops stemming from the pion poles. At the same time, chiral symmetry is preserved and a chiral counting scheme emerges. The considered Feynman diagrams contain two different heavy mass scales $M_{N}, M_{R}$ which we consider to satisfy the relation $M_{N}^{2} \ll M_{R}^{2}$. The utilized formalism is in general suited to study systems with two heavy mass scales in addition to a light mass scale. In this sense, it can be applied to other resonances as well, such as the $S_{11}(1535)$. In this case, however, an SU(3) calculation is necessary due to the important $\eta N$ decay channel.

\section{Acknowledgements}

We thank Jürg Gasser for an informative discussion on sigma terms and Siegfried Krewald for comments on the structure of the Roper. 


\section{References}

[1] S. Sasaki, T. Blum and S. Ohta, Phys. Rev. D 65 (2002) 074503 arXiv:hep-lat/0102010|.

[2] N. Mathur et al., Phys. Lett. B 605 (2005) 137 arXiv:hep-ph/0306199.

[3] D. B. Leinweber, W. Melnitchouk, D. G. Richards, A. G. Williams and J. M. Zanotti, Lect. Notes Phys. 663 (2005) 71 arXiv:nucl-th/0406032.

[4] D. Guadagnoli, M. Papinutto and S. Simula, Phys. Lett. B 604 (2004) 74 arXiv:hep-lat/0409011.

[5] K. Sasaki and S. Sasaki, Phys. Rev. D 72 (2005) 034502 arXiv:hep-lat/0503026.

[6] K. Sasaki, S. Sasaki and T. Hatsuda, Phys. Lett. B 623 (2005) 208 arXiv:hep-lat/0504020.

[7] T. Burch, C. Gattringer, L. Y. Glozman, C. Hagen, D. Hierl, C. B. Lang and A. Schäfer, Phys. Rev. D 74 (2006) 014504 arXiv:hep-lat/0604019.

[8] T. Becher and H. Leutwyler, Eur. Phys. J. C 9 (1999) 643 arXiv:hep-ph/9901384.

[9] N. Fettes, U.-G. Meißner, M. Mojžiš and S. Steininger, Annals Phys. 283 (2000) 273 [Erratum-ibid. 288 (2001) 249] arXiv:hep-ph/0001308.

[10] B. Borasoy and U.-G. Meißner, Annals Phys. 254 (1997) 192 arXiv:hep-ph/9607432.

[11] P. C. Bruns and U.-G. Meißner, Eur. Phys. J. C 40 (2005) 97 arXiv:hep-ph/0411223.

[12] J. Gasser and A. Zepeda, Nucl. Phys. B 174 (1980) 445.

[13] P. J. Ellis and H. B. Tang, Phys. Rev. C 57 (1998) 3356 arXiv:hep-ph/9709354.

[14] O. Krehl, C. Hanhart, S. Krewald and J. Speth, Phys. Rev. C 62 (2000) 025207 arXiv:nucl-th/9911080.

[15] V. Bernard, T. R. Hemmert and U.-G. Meißner, Phys. Lett. B 565 (2003) 137 arXiv:hep-ph/0303198.

[16] V. Bernard, T. R. Hemmert and U.-G. Meißner, Phys. Lett. B 622 (2005) 141 arXiv:hep-lat/0503022.

[17] C. Hacker, N. Wies, J. Gegelia and S. Scherer, Phys. Rev. C 72 (2005) 055203 arXiv:hep-ph/0505043.

[18] V. Bernard, N. Kaiser and U.-G. Meißner, Nucl. Phys. B 457 (1995) 147 arXiv:hep-ph/9507418.

[19] E. Hernandez, E. Oset and M. J. Vicente Vacas, Phys. Rev. C 66 (2002) 065201 arXiv:nucl-th/0209009.

[20] A. Holl, P. Maris, C. D. Roberts and S. V. Wright, arXiv:nucl-th/0512048

[21] I. P. Cavalcante, M. R. Robilotta, J. Sa Borges, D. de O.Santos and G. R. S. Zarnauskas, Phys. Rev. C 72 (2005) 065207 arXiv:hep-ph/0507147.

[22] V. Bernard, N. Kaiser and U.-G. Meißner, Nucl. Phys. A 615 (1997) 483 arXiv:hep-ph/9611253.

[23] G. Colangelo and S. Dürr, Eur. Phys. J. C 33 (2004) 543 arXiv:hep-lat/0311023.

[24] V. Bernard, T. R. Hemmert and U.-G. Meißner, Nucl. Phys. A 732 (2004) 149 arXiv:hep-ph/0307115.

[25] M. Procura, B. U. Musch, T. Wollenweber, T. R. Hemmert and W. Weise, Phys. Rev. D 73 (2006) 114510 arXiv:hep-lat/0603001. 